\title{
VIEWPOINT
}

\section{Intracranial Pressure Threshold Heuristics in Traumatic Brain Injury: One, None, Many!}

\author{
Christos Lazaridis ${ }^{1,2^{*}}$ (1), Masoom Desai ${ }^{3,4}$, George Damoulakis ${ }^{5}$ and Frederick A. Zeiler ${ }^{6,7,8,9}$
}

๑ 2020 Springer Science+Business Media, LLC, part of Springer Nature and Neurocritical Care Society

Critical care of the patient with severe traumatic brain injury (TBI) revolves around strategies to address intracranial hypertension (IHT), and optimizing cerebral perfusion. Strong evidence associates IHT (especially refractory IHT) with mortality, and unfavorable clinical outcomes. However, this association may merely indicate that IHT is a surrogate for severity of injury and not necessarily a modifiable, outcome-altering, treatment target, i.e., some patients die with IHT, not from IHT. Another reason for intracranial pressure (ICP), and derived cerebral perfusion pressure (CPP), to be central tenets of bedside management is the fact that are relatively easily monitored as opposed to other secondary brain injury (SBI) mechanisms such as metabolic and energy crisis, cortical spreading ischemia, diffusion microvascular hypoxia, and mitochondrial failure. Consequently, we may be misled targeting what we can measure, not necessarily what matters. A further concern is that by solely focusing on one pathophysiological aspect we may ignore complex multidimensional cascades leading to SBI, and our treatment interventions run the risk of being counterproductive and harmful. Despite limitations, it is unlikely that ICP-guided management should or will entirely go away in the near future. One telling indication is the concerns, and responses, generated by the Benchmark Evidence from South American Trials: Treatment of Intracranial Pressure (BEST TRIP) trial [1]. This is the only level-1 evidence-producing randomized clinical trial (RCT) on the topic, finding no primary outcome

\footnotetext{
*Correspondence: lazaridis@uchicago.edu

${ }^{1}$ Neurocritical Care Unit, Department of Neurology, University of Chicago, Medical Center, 5841 S. Maryland Avenue, Chicago, IL 60637, USA

Full list of author information is available at the end of the article

This comment refers to the article available at https://doi.org/10.1007/ s12028-020-00941-3.
}

difference among groups of TBI patients managed with ICP monitoring vs. an imaging and clinical exam (ICE) protocol. A consensus-of-experts conference was called to specifically alleviate a "misinterpretation" of the trial toward a nihilistic approach or an abandonment of ICP monitoring and targeted management [2].

In this viewpoint, we address the further issue of how to understand and decide upon the ICP threshold for intervention. We begin by a conceptual discussion of using physiologic thresholds as treatment-triggers in order to expose inherent flaws (and advantages) of thresholddriven protocols, and to contextualize assessment of the different approaches. A review follows, of merits and limitations of contemporary modes to ICP, namely the Brain Trauma Foundation (BTF) guidelines [3], the ICE protocol [4], and using multimodality neuromonitoring in order to individualize physiologic targets (Fig. 1 provides a summary). These perspectives roughly map to accepting a fixed and population-level ICP threshold, having no direct measurement and no ICP threshold, and having multiple, dynamic and individualized thresholds, respectively.

\section{What is a Threshold?}

Physiologic thresholds for treatment are constructs that involve clinical problem-solving heuristics. The development of some scientific concepts is not merely the result of applying scientific methods, it is also influenced by background theoretical perceptions, the state of technological advancement, and socioeconomic factors [5]. Pathophysiologic thresholds, as for ICP/CPP, are similarly conventions dictated by contemporary bedside medical technology, incomplete and oversimplified understanding of SBI mechanisms, and available medical treatments. A heuristic is any approach to problem-solving employing a practical method that is not guaranteed to be optimal, perfect or true, but which is nevertheless sufficient

\section{Springer}




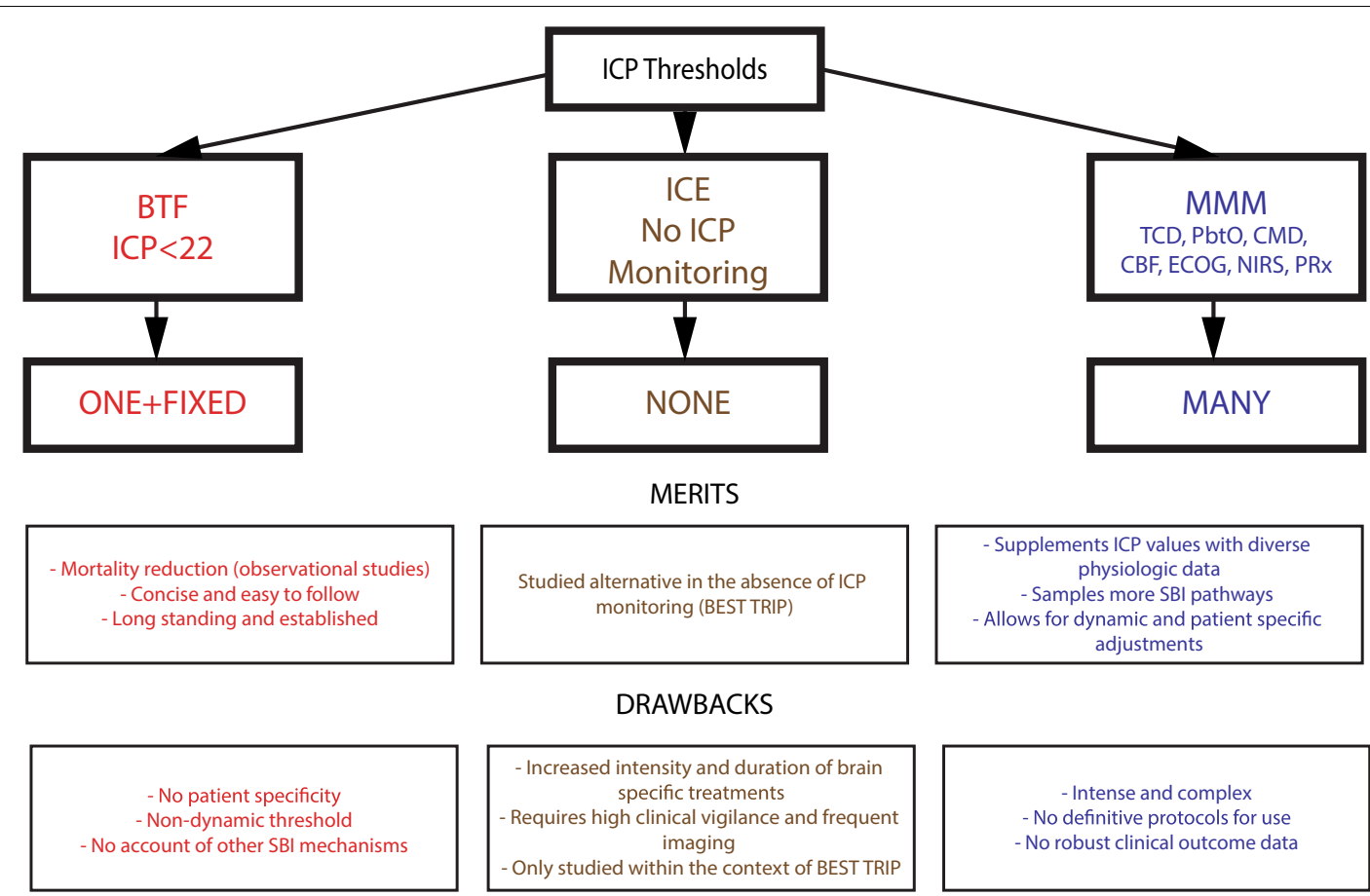

Fig. 1 Three contemporary approaches toward intracranial hypertension and pressure thresholds are depicted with respective merits and drawbacks. These approaches roughly map to adopting one, and fixed through time, ICP threshold ( $<22 \mathrm{mmHg}$ in the BTF guidelines), no ICP monitoring nor threshold in the ICE protocol, and the potential for variable and dynamic-through-time thresholds by employing multimodality monitoring. BEST TRIP Benchmark Evidence from South American Trials: Treatment of Intracranial Pressure trial, BTF Brain Trauma Foundation, CBF cerebral blood flow, CMD cerebral microdialysis, ECOG electrocorticography, ICE imaging and clinical exam, ICP intracranial pressure, MMM multimodality monitoring, NIRS near-infrared spectroscopy, $\mathrm{PbtO}_{2}$ partial brain tissue oxygen tension, $P R x$ pressure reactivity index, $S B /$ secondary brain injury, TCD transcranial Doppler

for reaching an immediate short-term goal. In fact, heuristics are strategies that ignore part of the information in order to make decisions faster, more frugally, or more accurately (a less-is-more effect) than more complex methods. Heuristics often operate as mental shortcuts that ease the cognitive load of making complex decisions. Violating an ICP threshold triggers a number of responses including obtaining imaging, adding therapies, changing tiers of ICP management, and overall altering clinical behaviors in protocolized or random ways. These alterations in clinical behavior, we suggest, are better understood as the clinical heuristics of responding to IHT that are inherent to threshold-guided management. As one reads on the different approaches below, another concept from the heuristics literature becomes relevant that of ecological rationality [6]. This relates to the idea that different strategies may be better suited for different clinical environments (better-not best-because in highly complex environments the optimal strategy can remain elusive).

\section{Brain Trauma Foundation Guidelines: Fixed Population-Level Threshold}

The BTF Guidelines have been widely incorporated into practice and associated with a reduction in mortality [7]. Plausibly, it is the protocolized approach as a whole that is of benefit rather than any of the individual components in isolation. Recently, a management algorithm that adopted the BTF recommendations for initial ICP/ CPP thresholds was proposed by the Seattle International Severe Traumatic Brain Injury Consensus Conference [8]. As a level IIB, the BTF has recommended an ICP threshold of $22 \mathrm{mmHg}$. This recommendation rests on a singlecenter study by Sorrentino et al. that accordingly to the BTF scored low in regards to quality-of-evidence [9]. The study was based on a 459-patient database (years 19922009) admitted with TBI to the Neuroscience Critical Care Unit at Addenbrooke's hospital, Cambridge, UK. The authors identified threshold values for ICP based on sequential chi-square distributions in which patients were dichotomized into survivors or not, and Glasgow Outcome Scale 1 to 3 versus 4 and 5; ICP was examined in steps of 1 in order to identify the level returning the highest chi-square score. The identified threshold was 
$22 \mathrm{~mm} \mathrm{Hg}$ for both mortality and favorable outcome across the entire cohort. When subgroups for age and sex were analyzed, the threshold did not change for mortality, but decreased to $18 \mathrm{mmHg}$ for favorable outcome for patients over 55 years of age, and women of all ages. Given that subgroup analysis may have been underpowered, and these findings are limited to only this study, the BTF did not support an ICP recommendation that varies by age or sex. Also, note that Sorrentino et al. included patients who underwent decompressive craniectomy (DC); it is known that DC reduces ICP (over 30\% of this population may have had a DC at some point during their stay in the given study), which may have impacted the described population-based thresholds [10, 11]. Furthermore, patients were under protocolized management with ICP thresholds in the 20-25 range, confounding the relationship of a threshold with outcomes in an actively treated group. Finally, this work utilized the entire recording period, and average ICP, for determination of epidemiologic thresholds. Recent evidence supports the notion that such population-based thresholds may vary over time [12].

The BTF approach is open to a number of criticisms. (a) The methods used do not allow differentiating between a potentially modifiable therapeutic target and a mere surrogate of severity. This is important since the ICP value is employed as a means of acting in order to alter an individual's clinical outcome; rather, what the study provides is a single population-level association of ICP with dichotomized outcomes. Thus, the methodology followed is vulnerable to an ecological fallacy, i.e., inferences about the nature of individuals are deduced from inferences about the group to which those individuals belong. The "group" here is patients with severe TBI; however, the assumption that this is a homogeneous sample of patients in terms of types of brain injuries, pathologies, and clinical trajectories, is erroneous [13, 14]. (b) SBI insults are treated as unidimensional excursions over a certain number, whereas degree and duration are not considered. (c) Pathophysiologic variables that describe the relationships among cerebral blood flow, perfusion pressure, oxygen delivery and utilization, as well as cellular metabolism and mitochondrial state remain unaccounted. (d) Therapeutic interventions carry adverse effects that could outweigh the benefits of achieving a given ICP goal [15]. Despite these limitations, a fixed threshold serves well the role of a successful heuristic by providing a consistent, and easy to follow by various experience and expertise providers, rule for acting; it is a "fast and frugal" heuristic model, which employs minimum time, knowledge, and computation to make adaptive choices in a real environment. Fast and frugal heuristics limit their search for information using easily pre-determined stopping rules (i.e., exceeding a certain ICP value for a certain period of time) and making choices with easily computable decision rules [16]. This is an important merit that has to be weighed against approaches that claim enhanced patient-specificity, or more accurate pathophysiology tracking, at the cost of increasing implementation complexity.

\section{Imaging Clinical Exam Protocol: No ICP Monitoring, No Threshold}

The ICE protocol was designed for the purposes of the BEST TRIP trial, and it directs the treatment of suspected IHT based on serial imaging and clinical examination; it is based on scheduled administration of hyperosmotic agents, with ancillary use of other treatments such as mild hyperventilation, and an escalating tiered approach. In BEST TRIP, the pre-specified primary outcome, as well as all other post hoc outcomes analyses, revealed no significant differences between groups (the control arm was ICP-monitored and managed along BTF lines). These results make the ICE protocol the only standard for the management of suspected IHT in the setting of TBI when ICP data are not available. One could further argue that the ICE protocol is an evidence-based alternative to invasive ICP-guided approaches. Nevertheless, external validity is questionable due to the special context of BEST TRIP in regards to pre-hospital resources, and post-intensive care unit (ICU) care. The concept of ecological rationality mentioned earlier is pertinent here, referring to the idea that the ICE protocol was developed according to the needs and experience in a specific clinical setting and may not be applicable to different healthcare systems.

Furthermore, although there was no difference between the ICP and ICE groups in terms of total length of ICU stay, the ICE group had significantly more ICU days involving brain-specific treatments (post hoc comparison also demonstrated a significantly greater number of interventions for the ICE group). There were no differences in terms of serious adverse events, frequency of neurosurgical procedures, or in the incidence of neurological worsening. An interpretation of these data suggests that lacking guidance from directly measured ICP requires high clinical vigilance, potentially frequent access to computed tomography and may lead to more brain-specific treatments both in terms of quantity and duration. Notwithstanding, it also shows that there is an alternative to the use of an ICP threshold as a trigger to act against IHT, and SBI; the ICE protocol is based on a different heuristic model, one that grounds anti-IHT tiered therapy on neurologic and radiographic assessments. 


\section{Patient-Specific Dynamic Thresholds}

TBI is highly heterogeneous manifesting in multiple phenotypes with differing pathophysiology, and clinical trajectories; it follows that using one fixed, generic threshold for all patients at all times is a gross oversimplification. However, difficult questions quickly arise. How should one go about individualizing, and adjusting through time physiologic thresholds? How multimodal should the approach be? How to integrate local with global physiologic data? What should one do when information from different monitors conflict or when there is dissociation between the clinical picture and tissue indices? What is the relationship between optimizing short-term physiologic tissue outcomes and longer-term clinical outcomes? Who are the patients to potentially benefit from such an approach? What is the availability of resources and expertise required in following patientspecific dynamic approaches? These are issues to be further explored before an individualizing approach becomes bedside clinical reality. It is important to note that this "many thresholds" approach appears to conflict with the initial definition of a heuristic. Here, the rules become more complex to follow; instead of mental shortcuts, this approach may lead to information overload, and complexity exceeding what a bedside clinician could safely handle.

The impetus for considering individualization, beyond the obvious heterogeneity of the disease, is related to the idea that the relationship of ICP with clinical outcome becomes qualified when it is investigated in conjunction with other SBI parameters such as the state of cerebrovascular pressure reactivity, quality of tissue oxygenation, and non-ICP-related metabolic and energy crisis. Some examples follow: patient-specific epidemiologic ICP thresholds in adult TBI have been derived by exploiting the relationship between the pressure reactivity index (PRx) and ICP, and displaying stronger association with mortality over fixed thresholds of 20 and $25 \mathrm{mmHg}$ (as a limitation note that individualized thresholds could not be obtained in about $1 / 3$ of the cohort) [17]. This outcome association held despite the fact that absolute ICP burden (as measured by dose of ICP, which integrates the degree of excursion over a certain threshold with the duration of the insult) was larger when calculated using the fixed vs. the PRx-derived thresholds [18]. In other words, the state of cerebrovascular pressure reactivity modified the impact of IHT on mortality, with impaired reactivity adding vulnerability across a wide range of ICP values. This result has been recently reproduced using data from the Collaborative European NeuroTrauma Effectiveness Research in TBI (CENTER-TBI) high-resolution ICU cohort (fixed thresholds of 20 and $22 \mathrm{mmHg}$ were used in the validation study) [19].
Multiple studies have shown dissociation between IHT and brain tissue hypoxia; the two insults can be concurrent or isolated. Observational cohorts have demonstrated that brain tissue hypoxia may occur even when ICP or CPP is normal and result from diffusion rather than perfusion defects $[20,21]$. Additionally, observational studies suggest that the addition of partial brain tissue oxygen tension $\left(\mathrm{PbtO}_{2}\right)$-directed care to conventional ICP/CPP-based management may be associated with improved outcome after severe TBI $[22,23]$. In the recent Brain Oxygen Optimization in Severe Traumatic Brain Injury Phase-II (BOOST II) trial, the trend toward more favorable outcomes and lower mortality with combined $\mathrm{ICP} / \mathrm{PbtO}_{2}$-guided treatment exceeded the pre-specified non-futility threshold, motivating the currently enrolling BOOST III [24]. The presence of potentially significant ischemic and/or hypoxic brain volume in patients with TBI with within-target ICP/CPP has been also evidenced using combined positron emission tomographymagnetic resonance imaging studies $[25,26]$. Similarly, non-ischemic metabolic crisis as monitored by cerebral microdialysis has been demonstrated in patients during active maintenance of $\mathrm{ICP}<20$ and $\mathrm{CPP}>60 \mathrm{mmHg}[27$, 28].

\section{Concluding Thoughts on Future Directions}

Intracranial hypertension is a common sequela after severe TBI and has consistently been associated with worse clinical outcomes. There remain unresolved questions in terms of making causal inferences, in unequivocally establishing ways of monitoring and managing, and on how to situate IHT within a wider framework of inter-related mechanisms and pathways contributing to SBI. Addressing heterogeneity should take high priority in the form of stratifying patients into subgroups with homogeneous pathophysiology, predicted risk for IHT, and expected outcome. Advances in multimodality neuromonitoring aspire to move treatments from a one-sizefits-all approach toward individualization. Specifically, we would benefit from models of physiologic latent states that can be used in mechanistic understanding, and outcome prediction. This requires exploration of high-quality large multivariate datasets comprised of continuous, real time physiologic data, and clinical/imaging characteristics to identify phenotypes of injury and patient-specific pathophysiological trajectories. Machine learning methodologies have been emerging with high accuracy in predicting episodes of IHT or brain tissue hypoxia; they do though require prospective validation [29-31]. In this overall direction, hugely important are ongoing collaborative projects such as Transforming Research and Clinical Knowledge in TBI (TRACK TBI), and CENTER-TBI. 
Methodological considerations are also relevant as controversies generated by BEST TRIP further highlight potential limitations of using traditional RCTs to evaluate monitoring devices and treatment thresholds; instead, we should consider comparative effectiveness research methodologies intending to measure differences in outcome and relate these both to the composite delivery of care and its components within, and across, specific clinical populations and settings.

\begin{abstract}
Author details
${ }^{1}$ Neurocritical Care Unit, Department of Neurology, University of Chicago, Medical Center, 5841 S. Maryland Avenue, Chicago, IL 60637, USA. ${ }^{2}$ Section of Neurosurgery, University of Chicago, Medical Center, Chicago, USA. ${ }^{3}$ Division of Neurocritical Care, University of Oklahoma Health Science Center, Oklahoma City, OK, USA. ${ }^{4}$ Division of Neurophysiology, University of Oklahoma Health Science Center, Oklahoma City, OK, USA. ${ }^{5}$ Department of Mechanical Engineering, University of Illinois at Chicago, Chicago, USA. ${ }^{6}$ Section of Neurosurgery, Department of Surgery, University of Manitoba, Winnipeg, Canada. ${ }^{7}$ Department of Human Anatomy and Cell Science, Rady Faculty of Health Sciences, University of Manitoba, Winnipeg, Canada. ${ }^{8}$ Biomedical Engineering, Faculty of Engineering, University of Manitoba, Winnipeg, Canada. ${ }^{9}$ Division of Anaesthesia, Department of Medicine, University of Cambridge, Cambridge, UK.
\end{abstract}

\section{Source of Support}

No funding has been received in relation to this manuscript.

\section{Conflicts of Interest}

None of the authors has conflicts of interest in relation to this manuscript.

\section{Publisher's Note}

Springer Nature remains neutral with regard to jurisdictional claims in published maps and institutional affiliations.

Published online: 5 March 2020

\section{References}

1. Chesnut RM, Temkin N, Carney N, et al. A trial of intracranial-pressure monitoring in traumatic brain injury. N Engl J Med. 2012;367:2471-81.

2. Chesnut RM, Bleck TP, Citerio G, et al. A consensus-based interpretation of the benchmark evidence from South American trials: treatment of intracranial pressure trial. J Neurotrauma. 2015:32:1722-4.

3. Carney N, Totten AM, O'Reilly C, et al. Guidelines for the management of severe traumatic brain injury. Neurosurgery. 2017;80:6-15.

4. Chesnut RM, Temkin N, Dikmen S, et al. A method of managing severe traumatic brain injury in the absence of intracranial pressure monitoring: the imaging and clinical examination protocol. J Neurotrauma. 2018;35(1):54-63.

5. Mallon R. Naturalistic approaches to social construction. In: Zalta EN, editor. The Stanford encyclopedia of philosophy (Spring 2019 Edition)

6. Gigerenzer G, Gaissmaier W. Heuristic decision making. Annu Rev Psychol. 2011;62:451-82.

7. Gerber LM, Chiu YL, Carney N, et al. Marked reduction in mortality in patients with severe traumatic brain injury. J Neurosurg. 2013;119:1583-90.

8. Hawryluk GWJ, Aguilera S, Buki A, et al. A management algorithm for patients with intracranial pressure monitoring: the Seattle International Severe Traumatic Brain Injury Consensus Conference (SIBICC). Intensive Care Med. 2019;45:1783-94.

9. Sorrentino E, Diedler J, Kasprowicz M, et al. Critical thresholds for cerebrovascular reactivity after traumatic brain injury. Neurocrit Care. 2012:16(2):258-66.
10. Timofeev I, Czosnyka M, Nortje J, et al. Effect of decompressive craniectomy on intracranial pressure and cerebrospinal compensation following traumatic brain injury. J Neurosurg. 2008;108(1):66-73.

11. Donnelly J, Czosnyka M, Adams H, et al. Twenty-five years of intracranial pressure monitoring after severe traumatic brain injury: a retrospective single-center analysis. Neurosurgery. 2019;85(1):E75-E82.

12. Nourallah B, Zeiler FA, Calviello L, et al. Critical thresholds for intracranial pressure vary over time in non-craniectomised traumatic brain injury patients. Acta Neurochir (Wien). 2018;160(7):1315-24.

13. Maas AIR, Menon DK, Adelson PD, et al. Traumatic brain injury: integrated approaches to improve prevention, clinical care, and research. Lancet Neurol. 2017;16(12):987-1048.

14. Jha RM, Elmer J, Zusman BE, et al. intracranial pressure trajectories: a novel approach to informing severe traumatic brain injury phenotypes. Crit Care Med. 2018;46(11):1792-802.

15. Kochanek PM, Tasker RC, Bell MJ, et al. Management of pediatric severe traumatic brain injury: 2019 consensus and guidelines-based algorithm for first and second tier therapies. Pediatr Crit Care Med. 2019;20(3):269-79.

16. Gigerenzer G, Hertwig R, Pachur T. Heuristics: the foundations of adaptive behavior. Oxford: Oxford University Press, Inc; 2011.

17. Lazaridis C, DeSantis SM, Smielewski P, et al. Patient-specific thresholds of intracranial pressure in severe traumatic brain injury. J Neurosurg. 2014;120(4):893-900.

18. Lazaridis C, Smielewski P, Menon DK, et al. Patient-specific thresholds and doses of intracranial hypertension in severe traumatic brain injury. Acta Neurochir Suppl. 2016;122:117-20.

19. Zeiler FA, Ercole A, Cabeleira M, et al. Patient-specific ICP epidemiologic thresholds in adult traumatic brain injury: a CENTER-TBI validation study. J Neurosurg Anesthesiol. 2019.

20. Menon DK, Coles JP, Gupta AK, et al. Diffusion limited oxygen delivery following head injury. Crit Care Med. 2004;32:1384-90.

21. Vespa PM, O'Phelan K, McArthur D, et al. Pericontusional brain tissue exhibits persistent elevation of lactate/pyruvate ratio independent of cerebral perfusion pressure. Crit Care Med. 2007;35:1153-60.

22. Bohman LE, Heuer GG, Macyszyn L, et al. Medical management of compromised brain oxygen in patients with severe traumatic brain injury. Neurocrit Care. 2011;14:361-9.

23. Nangunoori R, Maloney-Wilensky E, Stiefel M, et al. Brain tissue oxygenbased therapy and outcome after severe traumatic brain injury: a systematic literature review. Neurocrit Care. 2012;17:131-8.

24. Okonkwo DO, Shutter LA, Moore C, et al. Brain oxygen optimization in severe traumatic brain injury phase-II: a phase II randomized trial. Crit Care Med. 2017:45(11):1907-14.

25. Veenith TV, Carter EL, Geeraerts T, et al. Pathophysiologic mechanisms of cerebral ischemia and diffusion hypoxia in traumatic brain injury. JAMA Neurol. 2016;73(5):542-50.

26. Launey $Y$, Fryer TD, Hong YT, et al. Spatial and temporal pattern of ischemia and abnormal vascular function following traumatic brain injury. JAMA Neurol. 2019.

27. Vespa P, Bergsneider M, Hattori N, et al. Metabolic crisis without brain ischemia is common after traumatic brain injury: a combined microdialysis and positron emission tomography study. J Cereb Blood Flow Metab. 2005;25(6):763-74.

28. Stein NR, McArthur DL, Etchepare M, et al. Early cerebral metabolic crisis after TBI influences outcome despite adequate hemodynamic resuscitation. Neurocrit Care. 2012;17(1):49-57.

29. Güiza F, Depreitere B, Piper l, et al. Novel methods to predict increased intracranial pressure during intensive care and long-term neurologic outcome after traumatic brain injury: development and validation in a multicenter dataset. Crit Care Med. 2013;41(2):554-64.

30. Myers RB, Lazaridis $C$, Jermaine $C M$, et al. Predicting intracranial pressure and brain tissue oxygen crises in patients with severe traumatic brain injury. Crit Care Med. 2016;44(9):1754-61.

31. Güiza F, Depreitere B, Piper I, et al. Early detection of increased intracranial pressure episodes in traumatic brain injury: external validation in an adult and in a pediatric cohort. Crit Care Med. 2017;45(3):e316-e320320. 